Pacific Journal of Mathematics

SOME REMARKS ON ALGEBRAIC EQUIVALENCE OF
CYCLES 


\title{
SOME REMARKS ON ALGEBRAIC EQUIVALENCE OF CYCLES
}

\author{
Giuseppe Ceresa ANd Alberto Collino ${ }^{1}$
}

\begin{abstract}
Let $F \subseteq \mathbf{P}^{4}$ be a 3-fold with one ordinary double point $p$, and let $F^{\prime}$ be the proper transform of $F$ under the blowing up of $\mathrm{P}^{4}$ at $p$. If $H \subseteq F^{\prime}$ is the preimage of $p$ on $F^{\prime}$, we prove that for $F$ general the algebraic 1-cycle given by the difference of the two generators of the smooth quadric surface $H$, is not algebraically equivalent to zero on $F^{\prime}$. Griffiths has shown this cycle to be homologically equivalent to zero. Also, we show that on a general quintic 3 -fold $X$ there are no non-trivial algebraic equivalence relations between the lines of $X$.
\end{abstract}

One of the most remarkable results of Griffiths' paper on rational integrals [3] is the proof that homological equivalence does not imply algebraic equivalence for algebraic cycles. The argument is essentially based on two theorems, the so-called inversion theorem and the theorem of $\S 14$, stating properties of primitive cycles.

Our purpose here is to show that the inversion theorem alone implies, quite directly, that on a general threefold of degree 5 in $\mathbf{P}^{4}$ two lines are not algebraically equivalent, although they are homologically equivalent because of Lefschetz' theorem. Strengthening the inversion theorem a little bit we can also answer a natural question which may occur to a reader of [3] which we explain now. Let $F \subseteq \mathbf{P}^{4}$ be a threefold with exactly one singular point $p$, which is a node (ordinary double point) and let $F^{\prime}$ be the proper transform of $F$ under the blowing up of $\mathbf{P}^{4}$ at the node. $F^{\prime}$ is non-singular and the inverse image of $p$ is a smooth quadric surface $H$. If $L, M$, are two lines on $H$ belonging to the two different rulings then $L$ is homologically equivalent to $M$ on $F^{\prime}$, loc. cit. $\S 15$. The question is whether $L$ and $M$ are algebraically equivalent:

TheOREM. If $\operatorname{deg} F \geq 5$ and $F$ is general then $L$ and $M$ are not algebraically equivalent on $F^{\prime}$.

We thank H. Clemens for useful advice [1], which has allowed us to improve and simplify to a great extent a previous version of this paper.

\footnotetext{
${ }^{1}$ Both authors are members of G.N.S.A.G.A. of C.N.R.
} 


\section{The inversion theorem in the nodal case.}

(1.1) THEOREM. If $X \subseteq \mathbf{P}^{4}$ is a generic threefold with one node, of degree $d \geq 5$ and if $b: X^{\prime} \rightarrow X$ is the desingularization of $X$ obtained by blowing up the node, then every 1-cycle algebraically equivalent to 0 is contained in the kernel of the Abel-Jacobi map of $X^{\prime}$.

We recall that on a non-singular threefold $Y$ a class $\alpha \in H_{3}(Y, \mathbf{Z})$ is said to be of rank 2 if there is a surface $W$ and an inclusion $g: W \rightarrow Y$ such that $\alpha=g_{*}(\beta), \beta \in H_{3}(W, \mathbf{Z})$. Proposition 13.3 of [3] says that if there are no non-zero classes of rank 2 on $Y$ then the Abel-Jacobi map sends to 0 every cycle which is algebraically equivalent to 0 . To prove (1.1) it is enough to show

(1.2) If $X$ is as in (1.1) then $X^{\prime}$ contains no non-zero classes of rank 2.

REMARK. We stress that generic means that the set of threefolds which have non-zero classes of rank 2 is contained in a countable union of proper analytic subvarieties of the family $T$ of threefolds of degree $d$ with one node. In particular this implies that if in a pencil of threefolds one element has no non-zero classes of rank 2 then at most a countable number of elements in the pencil have non-zero classes of rank 2.

Proof of (1.1). For simplicity we set $d=5$. Let $\mathbf{P}^{N}$ be the projective space parametrizing the hypersurfaces of degree 5 in $\mathbf{P}^{4}$ and let $T$ be the subset of hypersurfaces with one node. Let $z_{0} \in T$ represent $X$ and let $D_{0}$ be its equation. Then:

(1.3) Locally at $z_{0} T$ is a non-singular hypersurface in $\mathbf{P}^{N}$. The tangent space to $T$ at $z_{0}$ is the hyperplane in $\mathbf{P}^{N}$ given by the lines through $z_{0}$ which correspond to pencils $D_{0}+\lambda E$, where $E$ is a polynomial of degree $d$ which satisfies the adjoint condition, namely $E$ passes through the node of $D_{0}$.

The proof of this fact is elementary and we omit it.

Now, fix $\alpha \in H_{3}\left(X^{\prime}, \mathbf{Z}\right)$ and suppose that there is a neighborhood $U$ of $z_{0}$ in $T$ containing a dense subset $U^{*}$ with the property that for $z \in U^{*}$ the cycle $\alpha_{z}$ is of rank 2 on $X_{z}^{\prime}$. By $\alpha_{z}$, we mean the cycle class obtained by the following process. Choose a representative cycle for $\alpha$ on $X$ which does not pass through the node, (such a representative exists by (15.9) of [3]), 
and transport it to nearby $X_{z}$ by taking a solid tube over this representative and intersecting it with $X_{z}$, see loc. cit. $\S 3$. If $X_{z}$ is sufficiently close to $X$, then the transported cycle does not meet the node on $X_{z}$. The lifting of this cycle to $X_{z}^{\prime}$ is what we call $\alpha_{z}$. Note that $\alpha_{z}$ of rank 2 implies that there exists a surface $W_{z}$ and an inclusion map $g_{z}: W_{z} \rightarrow X_{z}^{\prime}$ such that $\alpha_{z}=$ $\left(g_{z}\right)_{*}\left(\beta_{z}\right)$. Following the notation of [3], set $\eta_{z}=\Omega / D_{z}$, where $D_{z}$ is the equation of $X_{z}$, and let $b_{z}:\left(\mathbf{P}^{4}\right)^{\prime} \rightarrow \mathbf{P}^{4}$ be the blowing up of $\mathbf{P}^{4}$ at the node of $X_{z}$. By loc. cit. $\S 16$, the residue $R\left(b_{z}^{*} \eta_{z}\right)$ induces a cohomology class in $H^{3,0}\left(X_{z}^{\prime}\right)$. Then

$$
\int_{\alpha_{z}} R\left(b_{z}^{*} \eta_{z}\right)=\int_{\beta_{z}} g_{z}^{*} R\left(b_{z}^{*} \eta_{z}\right)=0
$$

because on a surface every $(3,0)$ form is 0 .

Also, since the integral (1.4) is an analytic function of $z$ vanishing on $U^{*}$, it is identically zero on $U$.

Let $D(\lambda)=\sum_{k \geq 0} D_{k}\left(x_{0}, \ldots, x_{4}\right) \lambda^{k}$ be an analytic curve on $T$ centered at $D_{0}$, and suppose that for small $|\lambda| D(\lambda)$ is the equation of a threefold $X_{\lambda}$ with one single node so that the corresponding point $z(\lambda)$ is in $U$. By (1.3) the polynomial $D_{1}$ satisfies the adjoint condition, and conversely every polynomial which satisfies the adjoint condition can be given as $D_{1}$ in the power series expansion of some $D(\lambda)$.

The integral (1.4) is then a function of $\lambda$ which is identically 0 because $z(\lambda) \in U$. Differentiating (1.4) at $\lambda=0$ gives (see [3], pg. 508)

$$
\left.0=\int_{\alpha} R\left(b_{o}^{*}\left(-D_{1} \Omega / D_{0}^{2}\right)\right) \quad \text { where } R b^{*}\left(D_{1} \Omega / D_{0}^{2}\right)\right) \in F^{2} H^{3}\left(X^{\prime}\right) .
$$

Thus $\int_{\alpha} \omega=0$ for every $\omega \in F^{2} H^{3}\left(X^{\prime}\right)$, because this vector space is generated by residues of type (1.5) (see loc. cit. §16). Since $\alpha$ is a real homology class, $0=\int_{\alpha} \omega=\int_{\alpha} \bar{\omega}$, hence by Hodge's theorems $\alpha=0$.

We have therefore proved that if $0 \neq \alpha \in H_{3}\left(X^{\prime}, \mathbf{Z}\right)$ then there is a neighborhood $U$ of $z_{0}$ in $T$ such that the set of points $z \in U$ for which $\alpha_{z}$ is of rank 2 is a proper analytic subvariety of $U$. The statement in (1.2) follows by varying $\alpha$ in $H_{3}\left(X^{\prime}, \mathbf{Z}\right)$.

II. The nodal case. Let $G=Q\left(x_{0}, \ldots, x_{3}\right) x_{4}^{d-2}+K\left(x_{0}, \ldots, x_{3}\right)$ be the homogeneous equation of a threefold $V_{0}$ in $\mathbf{P}^{4}$ of degree $d$. We assume that $K$ and $Q$ are the equations of two non-singular surfaces in $\mathbf{P}^{3}$ which intersect transversally along a curve $C$. Then $V_{0}$ is non-singular, but for the node at $p=(0,0,0,0,1)$. Blowing up $V_{0}$ at $p$ yields $V_{0}^{\prime}$, and the linear projection from $p$ induces a morphism $g: V_{0}^{\prime} \rightarrow B_{C}\left(\mathbf{P}^{3}\right)$, where $B_{C}\left(\mathbf{P}^{3}\right)$ is 
the blowing up of $\mathbf{P}^{3}$ along $C$. The map $g$ is a finite covering of degree $(d-2)$, and the exceptional divisor $H$ on $V_{0}^{\prime}$ is mapped isomorphically onto $Q^{\prime}$, the proper transform on $B_{C}\left(\mathbf{P}^{3}\right)$ of the quadric $Q$.

Since the Jacobian variety of $C$ and the intermediate Jacobian of $B_{C}\left(\mathbf{P}^{3}\right)$ are isomorphic [2], the morphism of intermediate Jacobians $g_{*}$ : $J\left(V_{0}^{\prime}\right) \rightarrow J\left(B_{C}\left(\mathbf{P}^{3}\right)\right)$ induces a morphism $g_{*}: J\left(V_{0}^{\prime}\right) \rightarrow J(C)$. A straight computation gives

$$
g_{*}(\varphi(L-M))=-i^{*}(L-M) \text { in } J(C),
$$

where $\varphi$ is the Abel-Jacobi map, $i: C \rightarrow Q$ is the inclusion, $i^{*}: \operatorname{Pic}(Q) \rightarrow$ $\operatorname{Pic}^{0}(C) \simeq J(C)$, and $L, M$ are lines representing the two different rulings of $Q \simeq Q^{\prime} \simeq H$.

(2.2) LEMMA. $\varphi(L-M) \neq 0$ in $J\left(V_{0}^{\prime}\right)$.

Proof. It suffices to show that $i^{*}(L-M)$ is not trivial in $\operatorname{Pic}(C)$. If it were, the first ruling on $Q$ would cut on $C$ a linear system which would not be complete, because $i^{*}(M)$ is not cut by the first ruling. On the other hand, it is easy to show that the first ruling cuts on $C$ a complete linear system.

Now, let $V$ be a generic threefold of degree $d$ with one single node. Without restriction we may assume that the equation of $V$ is $F\left(x_{0}, \ldots, x_{4}\right)$ $=Q x^{d-2}+\cdots=0$, i.e., $V$ is singular at $p$ and it has the same tangent cone as $V_{0}$. Define a fourfold $\mathscr{V} \subseteq \mathbf{P}^{1} \times \mathbf{P}^{4}$ by the equation $s F+t G=0$ and blow it up along $\mathbf{P}^{1} \times\{p\}$ to obtain a family $\pi: \mathcal{V}^{\prime} \rightarrow \mathbf{P}^{1}, \pi^{-1}((0,1))$ $=V_{0}$. Note that the exceptional divisor on $\mathcal{V}^{\prime}$ is isomorphic to $\mathbf{P}^{1} \times H$, so that on $V_{s}^{\prime}=\pi^{-1}((s, 1))$ the exceptional divisor is identified with $H$, the exceptional divisor on $V_{0}^{\prime}$.

Set $B=\left\{z \in \mathbf{P}^{1}: V_{z}^{\prime}\right.$ is non-singular $\}$, and let $\mathcal{G} \rightarrow B$ be the associated family of intermediate Jacobians.

Fixing $L$ and $M$ on $H$, the family of cycles $\left(\mathbf{P}^{1} \times L-\mathbf{P}^{1} \times M\right)$ gives a section $\sigma: B \rightarrow \mathcal{G}$, defined by means of the Abel-Jacobi maps, i.e., $\sigma(z)=\varphi_{z}(L-M) \in J\left(V_{z}^{\prime}\right)$.

(2.3) LEMMA. $\sigma$ is not identically zero.

Proof. By $(2.2) \sigma(0) \neq 0$ and $\sigma$ is analytic.

By our choice of $V_{\infty}^{\prime}=V^{\prime}$ and the remark after (1.2), for generic $z \in B$ the kernel of the Abel-Jacobi map $\varphi_{z}$ contains all the cycles which 
are algebraically equivalent to zero on $V_{z}^{\prime}$; on the other hand, by the lemma $0 \neq \sigma(z)=\varphi_{z}(L-M)$, hence

(2.4) For generic $z$ the lines $L$ and $M$ are not algebraically equivalent on $V_{z}^{\prime}$.

III. Lines on a quintic threefold. We recall that on a generic non-singular threefold of degree 5 there are at least two lines which do not intersect [4]; we shall show that they are not algebraically equivalent. Since the method of the proof is the same as in the nodal case, we only construct the analogue of $V_{0}^{\prime}$ and leave further details to the reader.

Our purpose is to produce a smooth quintic threefold $W$ containing two lines, $l_{a}$ and $l_{b}$, which do not intersect and such that the cycle $\left(l_{a}-l_{b}\right)$ does not belong to the kernel of the Abel-Jacobi map $\varphi$. For this we consider the threefold $W$ defined by the equation $x_{0} x_{4}^{4}+$ $K\left(x_{0}, \ldots, x_{3}\right)=0$, where: (i) $K$ is a non-singular surface in $\mathbf{P}^{3}$ of degree 5, (ii) $K$ contains two lines $l_{a}^{*}$ and $l_{b}^{*}$ which do not intersect and do not lie on the plane $H:\left\{x_{0}=0\right\}$, (iii) in $\mathbf{P}^{3}, H$ and $K$ intersect transversally along a curve $C$. Then, on $W$, the lines $l_{a}$ and $l_{b}$ are the lines $l_{a}^{*}, l_{b}^{*}$ contained in the hyperplane section $x_{4}=0=K$.

Blowing up $W$ at $p=(0,0,0,0,1)$ gives $W^{\prime}$, and we have $J(W) \simeq$ $J\left(W^{\prime}\right)$ and $\varphi\left(l_{a}-l_{b}\right)=\varphi\left(l_{a}^{\prime}-l_{b}^{\prime}\right)$, where $l^{\prime}$ denotes the proper transform of $l$ on $W^{\prime}$.

As in (2.1) we get a morphism $g_{*}: J\left(W^{\prime}\right) \rightarrow J(C)$ and $g_{*}\left(\varphi\left(l_{a}-l_{b}\right)\right)$ $=-\operatorname{class}\left(z_{a}-z_{b}\right)$, where $z_{a}, z_{b} \in C$ are the points of intersections of $l_{a}$ and $l_{b}$ with $H$.

(3.1) LEMMA. $\varphi\left(l_{a}-l_{b}\right) \neq 0$.

Proof. It suffices to show that on $C z_{a}$ and $z_{b}$ are not linearly equivalent. This is true since $C$ is a plane curve of degree $>3$, hence not hyperelliptic.

Arguing as in the nodal case one has

(3.2) Two lines on a general quintic threefold are not algebraically equivalent.

Also we thank the referee for suggesting to us this more general statement. 
(3.3) If $X$ is a general quintic threefold, $l_{1}, \ldots, l_{2875}$ the lines on $X$, then no linear combination

$$
\sum a_{i} l_{i}
$$

of the $l_{i}$ is algebraically equivalent to zero.

The reason is that if we have a relation $\sum a_{i} l_{i} \sim 0$, it would follow that $\sum a_{i} l_{\sigma(l)} \sim 0$ for any $\sigma$ in the monodromy group $M$ of the 2875 lines. Since $M=S_{2875}$, then, any relation at all would imply that $l_{i} \sim l_{j}$ for all $i, j$.

\section{REFERENCES}

1. C. H. Clemens, Applications of mixed Hodge theory to the study of threefolds, Rend. Sem. Mat. Univ. Politecn. Torino, 39, 1 (1981).

2. C. H. Clemens and P. Griffiths, The intermediate Jacobian of the cubic threefold, Ann. of Math., 95 (1972), 281-356.

3. P. Griffiths, On the period of certain rational integrals, I and II, Ann. of Math., 90 (1969), 460-541.

4. J. Harris, Galois groups of enumerative problems, Duke Math. J., 46 (1979), 685-724.

Received September 25, 1981.

UNIVERSITA DI TORINO

ViA P. AMEDEO 8

10123 TORINO, ITALY 


\title{
PACIFIC JOURNAL OF MATHEMATICS EDITORS
}

\author{
Donald BabBitT (Managing Editor) \\ University of California \\ Los Angeles, CA 90024 \\ Hugo Rossi \\ University of Utah \\ Salt Lake City, UT 84112 \\ C. C. Moore and Arthur Ogus \\ University of California \\ Berkeley, CA 94720
}

J. DugundiI

Department of Mathematics

University of Southern California

Los Angeles, CA 90089-1113

R. FINN and H. SAMELSON

Stanford University

Stanford, CA 94305

\section{ASSOCIATE EDITORS}
R. ARENS
E. F. BECKENBACH
B. H. NeUMANN
F. WOLF
K. YosHIDA

(1906-1982)

\section{SUPPORTING INSTITUTIONS}

UNIVERSITY OF ARIZONA

UNIVERSITY OF BRITISH COLUMBIA

CALIFORNIA INSTITUTE OF TECHNOLOGY

UNIVERSITY OF CALIFORNIA

MONTANA STATE UNIVERSITY

UNIVERSITY OF NEVADA, RENO

NEW MEXICO STATE UNIVERSITY

OREGON STATE UNIVERSITY

\author{
UNIVERSITY OF OREGON \\ UNIVERSITY OF SOUTHERN CALIFORNIA \\ STANFORD UNIVERSITY \\ UNIVERSITY OF HAWAII \\ UNIVERSITY OF TOKYO \\ UNIVERSITY OF UTAH \\ WASHINGTON STATE UNIVERSITY \\ UNIVERSITY OF WASHINGTON
}

The Supporting Institutions listed above contribute to the cost of publication of this Journal, but they are not owners or publishers and have no responsibility for its content or policies.

Mathematical papers intended for publication in the Pacific Journal of Mathematics should be in typed form or offset-reproduced (not dittoed), double spaced with large margins. Please do not use built up fractions in the text of the manuscript. However, you may use them in the displayed equations. Underline Greek letters in red, German in green, and script in blue. The first paragraph must be capable of being used separately as a synopsis of the entire paper. In particular it should contain no bibliographic references. Please propose a heading for the odd numbered pages of less than 35 characters. Manuscripts, in triplicate, may be sent to any one of the editors. Please classify according to the scheme of Math. Reviews, Index to Vol. 39. Supply name and address of author to whom proofs should be sent. All other communications should be addressed to the managing editor, or Elaine Barth, University of California, Los Angeles, California 90024.

There are page-charges associated with articles appearing in the Pacific Journal of Mathematics. These charges are expected to be paid by the author's University, Government Agency or Company. If the author or authors do not have access to such Institutional support these charges are waived. Single authors will receive 50 free reprints; joint authors will receive a total of 100 free reprints. Additional copies may be obtained at cost in multiples of 50 .

The Pacific Journal of Mathematıcs is issued monthly as of January 1966. Regular subscription rate: $\$ 132.00$ a year (6 Vol., 12 issues). Special rate: $\$ 66.00$ a year to individual members of supporting institutions.

Subscriptions, orders for numbers issued in the last three calendar years, and changes of address should be sent to Pacific Journal of Mathematics, P.O. Box 969, Carmel Valley, CA 93924, U.S.A. Old back numbers obtainable from Kraus Periodicals Co., Route 100, Millwood, NY 10546.

The Pacific Journal of Mathematics ISSN 0030-8730 is published monthly by the Pacific Journal of Mathematics at P.O. Box 969, Carmel Valley, CA 93924. Application to mail at Second-class postage rates is pend ing at Carmel Valley, California, and additional mailing offices. Postmaster: Send address changes to Pacific Journal of Mathematics, P. O. Box 969, Carmel Valley, CA 93924.

PUBLISHED BY PACIFIC JOURNAL OF MATHEMATICS, A NON-PROFIT CORPORATION

Copyright $(1) 1983$ by Pacific Journal of Mathematics 


\section{Pacific Journal of Mathematics}

\section{Vol. 105, No. $2 \quad$ October, 1983}

Spiros Argyros, On compact spaces without strictly positive measure . . . . . 257

Steven Robert Bell, Regularity of the Bergman projection in certain nonpseudoconvex domains ............................273

Carlos R. Borges and Gary Fred Gruenhage, Sup-characterization of

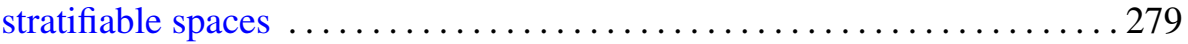

Giuseppe Ceresa and Alberto Collino, Some remarks on algebraic

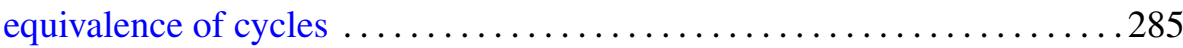

Charles Kam-Tai Chui and Maurice Hasson, Degree of uniform approximation on disjoint intervals ....................... 291

Gary Gundersen, Meromorphic functions that share two finite values with

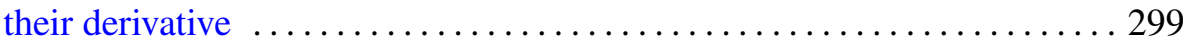

Richard I. Hartley, Lifting group homomorphisms ............... 311

Gerald William Johnson and David Lee Skoug, Notes on the Feynman integral. III. The Schroedinger equation ..................... 321

John Cronan Kieffer, Some topologies on the set of discrete stationary

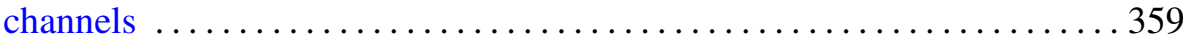

Harald Luschgy and Wolfgang Thomsen, Extreme points in the Hahn-Banach-Kantorovič setting . ............................... 387

Zbigniew Piotrowski, A. Rosłanowski and Brian M. Scott, The pinched-cube topology ................................ 399

Elias Saab and Paulette Saab, A dual geometric characterization of Banach

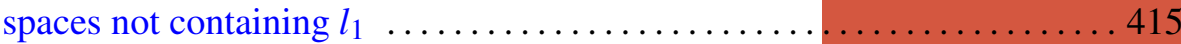

Walter Schachermayer, Norm attaining operators on some classical Banach

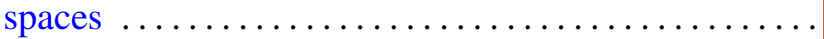

Martin Scharlemann, Essential tori in 4-manifold boundaries

Jacques C. H. Simon, Nonlinear representations of Poincaré group and global solutions of relativistic wave equations ..........

Adrian R. Wadsworth, $p$-Henselian field: $K$-theory, Galois cohomology, and graded Witt rings $\ldots \ldots \ldots \ldots \ldots \ldots \ldots \ldots \ldots$ 